\title{
DSURVEY: A BLOCKCHAIN-ENHANCED SURVEY PlATFORM FOR THE DATA ECONOMY
}

\author{
Alessio Bonti, Akanksha Saini, Thien Pham, \\ Mohamed Abdelrazek and Lorenzo Pinto
}

Deakin University, Burwood, Melbourne, Victoria, Australia

\begin{abstract}
The data economy is predicted to boom and become a $156 \mathrm{~B}$ dollars business by 2025. In this demo we introduce the use of distributed ledger technologies (DLT) applied to digital surveys in order to create an ecosystem where data becomes a central piece of a complex economy. Our system allows for interesting key features; ownership, traceability, secure profiles, and anonymity where required. Also, the most important feature, is the incentive mechanism that rewards all participants, both users creating surveys and those answering the surveys. DSurvey (decentralized survey) is a novel application framework that aims at moving away from the large commercial data sink paradigm whose business is restricted to gathering data and reselling it. Our solution makes so that no central data sink exists, and it always belongs to the creator, who are able to know who is using it, and receive royalties.
\end{abstract}

\section{KEYWORDS}

Decentralized survey, data ownership, incentive mechanism, ICO, blockchain.

\section{INTRODUCTION}

Over the past years, many organizations, businesses and researchers have been conducting online surveys to have an unbiased approach to decision making about their products and services. It helps them to understand their business and develop decision making. This not only helps to gather data but also makes the organizations learn about the customer's meaningful opinions, comments, and feedback. Survey applications have advantages in terms of collecting unbiased results. These results contribute to set benchmarks for comparisons required to perform over time. The time needed, the engagement, response time, low costs and real time access are just some of the many other advantages that digital surveys have over paper ones.

On the other hand, these techniques face challenges in securing a person's identity since most of the surveys require participants to reveal sensitive information about them. Another limitation and challenge are restricting the same person from doing the survey many times, sometimes termed as survey fraud [1]. The intention of the survey fraud is to get the incentives without contributing towards the advancement of the study [1]. Also, online surveys are usually conducted by a centralized entity, which turns out to be a single point of failure or ownership. A huge amount of response data and identity information is stored and controlled by this, which can further use the data for analysis or possibly to manipulate for malicious activities.

In addition, data has been considered as a tradeable commodity which companies can sell for monetary profits (data monetization). Furthermore, data-driven companies have to collect data to gain competitive advantages, the more valuable data they have, the more advantages they will

David C. Wyld et al. (Eds): AIAP, SIGML, CNSA, NIAI - 2021

pp. 177-194, 2021. CS \& IT - CSCP 2021

DOI: $10.5121 /$ csit.2021.110112 
have over other competitors [2]. Besides, users' awareness of the value of their personal data has been reasonably increasing after a few incidents, especially after the case of FacebookCambridge Analytica [3]. Regulations and legislation have been issued by governments around the world in order to adapt with the rapid growth of data economy, not only to control the economy, but also to protect users' personal data against malicious activities [3]. It is predicted in 2016 that by 2025 , the data economy will be worth 156B dollars. However, the United Nations reported the total economic impact of EU's data economy was more than 335 billion euros [3] which dramatically exceeded the prediction of IBM in [2]. The world's top 3 data rich companies are reported as General Electric, IBM, and Amazon [4] who have all together invested nearly 3 billion AUD in the data economy in order to get real-time and accurate analysis with higher profits and sustained traceability without human intervention [2].

In order to create a strong foundation for the future of data, a paradigm shift in data ownership and privacy must be made. With the increasing use of Distributed AI, data should not be allowed to come from large solitary data sinks, instead, data should always be traceable (provenance) and distributed. To allow for this and more, we have based our proposed system on blockchain. DSurvey is a decentralized digital survey platform complete with its cryptocurrency. It allows users to generate and answer surveys, allocating bounties to these so to create an incentive-based mechanism, in contrast with commercially available products which acquire both data privacy and ownership. It is an application designed for every person who wishes to provide personal data in exchange for a fee and at the same time, retain ownership of it. We are revolutionizing the economy of data. As people own and license it, we can imagine a future with more possibilities, as we all know, data is the next gold. DSurvey is one of a line of applications that aims at moving data ownership from large data sinks such as commercial survey platforms. It uses a welldesigned architecture based on the use of blockchain. In our implementation, we make use of Hyperledger Fabric [5], mainly due to its very high performance in comparison to other distributed ledger solutions.

The remainder of this paper is organized as follows. Section 2 discusses the motivations driving the development of DSurvey and its promising contribution to the current pain points, Section 3 presents the proposed decentralised survey system architecture and explains the framework and working of our proposed model. Section 4 highlights our security model and KYC process. We detail the corresponding design and implementation in Section 5. Section 6 presents the discusses the proposed system and concludes the paper.

\section{Motivation AND CONTRibution}

\subsection{Motivation}

The motivation of creating DSurvey comes from two primary points: the promising profit offered by data economy and the revolution of data ownership.

As mentioned above, the data economy has been showing the promising future of becoming a multi-billion dollars economy. However, as the economy is growing, there are more competitions between companies, thus, data is generated at an incredible speed. Hence, high quality data which is worth investing takes considerable efforts to generate. In the world where data amount is overwhelming, high quality data has to be the one that novel, reliable and specific enough, so that other companies may consider investing their money to access to the results directly instead of regenerating a new one themselves. 
However, generating high quality data through surveys is another challenge. Since online surveys have $11 \%$ lower response rates comparing to other types, according to [6-8] cited in [1]. Also, people have become more reluctant to participate in surveys due to the risk of losing their privacy and the uncertainty of their ownership over their own data. Since they do not know how their data will be used and who is it going to be shared with. Despite multitude of actions have been taken by the governments around the world to control the use of data, yet trust is still not regained. In [9], people were claimed to rather build a direct relation with companies to share their data as they did not think government's regulation could protect their data.

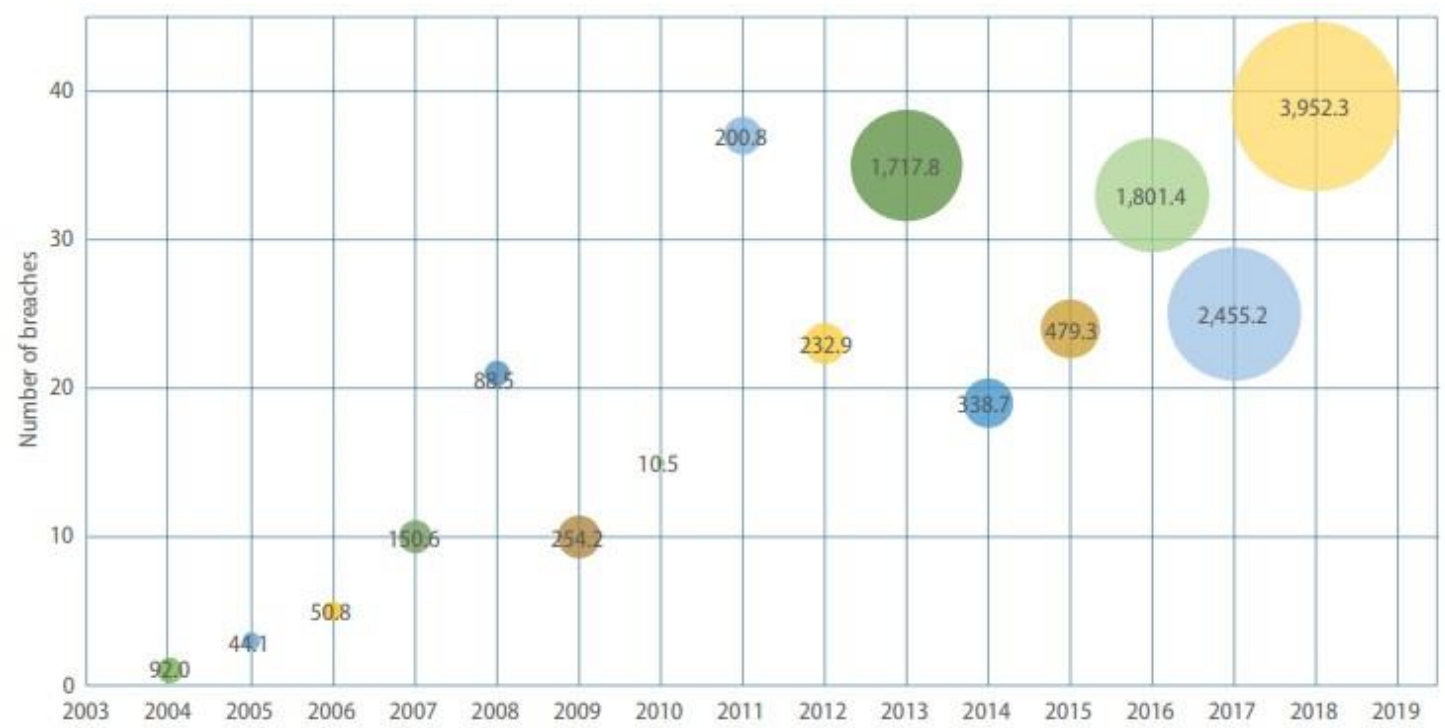

Figure 1. Number of data breaches and number of records exposed (indicated within the bubbles in millions), by year [3].

\subsection{Contribution}

Blockchain appears to be an emerging technology that will help both data collectors regain trust from end-users and data subjects to provide their data with great confidence. Blockchain is a technology that boomed with the release of bitcoin [10] and offers many advantages over standard databases. It allows the system we have implemented to be decentrally controlled, be tamper proof and provide an always up-to-date traceability of the data recorded [11], which is the base of data ownership. Since, we are working within a collaborative and hostile environment, traceability is a key requirement so that stakeholders can trust each other. Using blockchain, one cannot alter the data and create a new state without notifying others [11]. Hence, using blockchain creates a system of implicit trust.

By applying blockchain into our proposed system, it is expected to firstly regain trust from individuals, so that they can confidently share their data with data collectors, by providing them with data privacy and data ownership. Secondly, the system aims to replace current online surveys with decentralised surveys by offering more data security, less data sharing efforts and ease of data retrieving. Furthermore, response rates are also expected to be improved in the proposed system by the implementation of our incentives mechanism [1], which is taken place on the secured and transparent network of blockchain.

Trust is regained from end-users by offering them genuine data ownership and guarantee of their data privacy. Surveyees will be able to know where their data goes and decide to give consent for 
data access every time the data is requested to be obtained. This is allowed by the implementation of consensus rules and chaincodes which in turns defines transaction rules and access controls over the data within the Hyperledger Fabric network. Apart from offering chaincodes, as smart contracts, to be the valid decentralized application with security features from blockchain and its consensus among network peers by which the business logic of the blockchain application is defined. Hyperledger Fabric also provide business with the ability to customize their consensus rules to define expected and valid actions throughout the network [5]. Transactions on the blockchain network are non-repudiable [12], which means data within our network is protected against unauthorized access and untrustworthy sharing between companies. Moreover, DSurvey system facilitates data sharing with ease and reduce cost and time for data retrieval, since data of different entities on the network can be integrated into blockchain system instead of separately stored individually on each system [13].

DSurvey is based on its own cryptocurrency, the DScoin, created through an Initial Coin Offering (ICO) distributed among the verified clients. This is used to generate a service-based economy. In order to increase response rates of DSurvey, we have implemented the incentive mechanism [1] based on the DScoin, which does not only attract data subjects (individuals), but also create an economy-like environment for data exchanging between those who collect it. To this extent we have taken into consideration, the most commonly used reward mechanism that are compatible with online practices, these are bounties, royalty fees and early client (or early adopters) mechanisms.

\section{SURVEY FRAMEWORK}

\subsection{Decentralized Survey Architecture}

In the following, we will differentiate clients as $\mathrm{C}$ and participants as $\mathrm{P}$, a client can be identified as a company that would like to create surveys, and $\mathrm{P}$ as participants that want to answer surveys, within the system, they all logically appear as users and have the same roles. As illustrated in Figure 2, the proposed framework consists of multiple clients $(\mathrm{C} 1, \mathrm{C} 2, \mathrm{C} 3 \ldots \ldots ., \mathrm{Cn})$ each of them can own and create surveys, and participants (P1, P2, P3 ..... Pn) who participate in conducting the survey. The system is managed by a decentralized survey platform storing all the entries of clients and participants involved in the system. An Initial Coin Offering (ICO) has been built and distributed a portion of crypto tokens to the new joining clients as a way for their investment. Once the ICO is over, our system gets started. Participants are rewarded with some coins as incentive from the client after completing the corresponding survey. The following is a list of all building blocks in the system:

\subsubsection{Decentralized survey application:}

DSurvey is a decentralized survey application, it manages a list of clients and participants on Sovrin - public permissioned ledger [12]. All the clients and participants are verified before entering the system. It offers an ICO to initiate the process. DSurvey is based on a permissioned ledger, this allows for our system to have a higher throughput while still achieving a high level of trust because validator nodes are operated by trusted parties. Although the permissioned ledger does not fully reflect an independent blockchain network such as Ethereum, it still allows us to provide the key characteristics that describe a blockchain based system, immutability, trust, and traceability. A new client is only added into the system after being verified by the Sovrin service. 


\subsubsection{Clients:}

Clients are designated as "C" in our system. They make requests to join the system and upload the survey required to be carried out. They can read the responses from the participants.

\subsubsection{Participants:}

Participants are represented as "P" in our system. Various participants wish to subscribe to the specific survey through the Sovrin ledger. They can read the survey and give their viewpoints without increasing the load on the validator node.

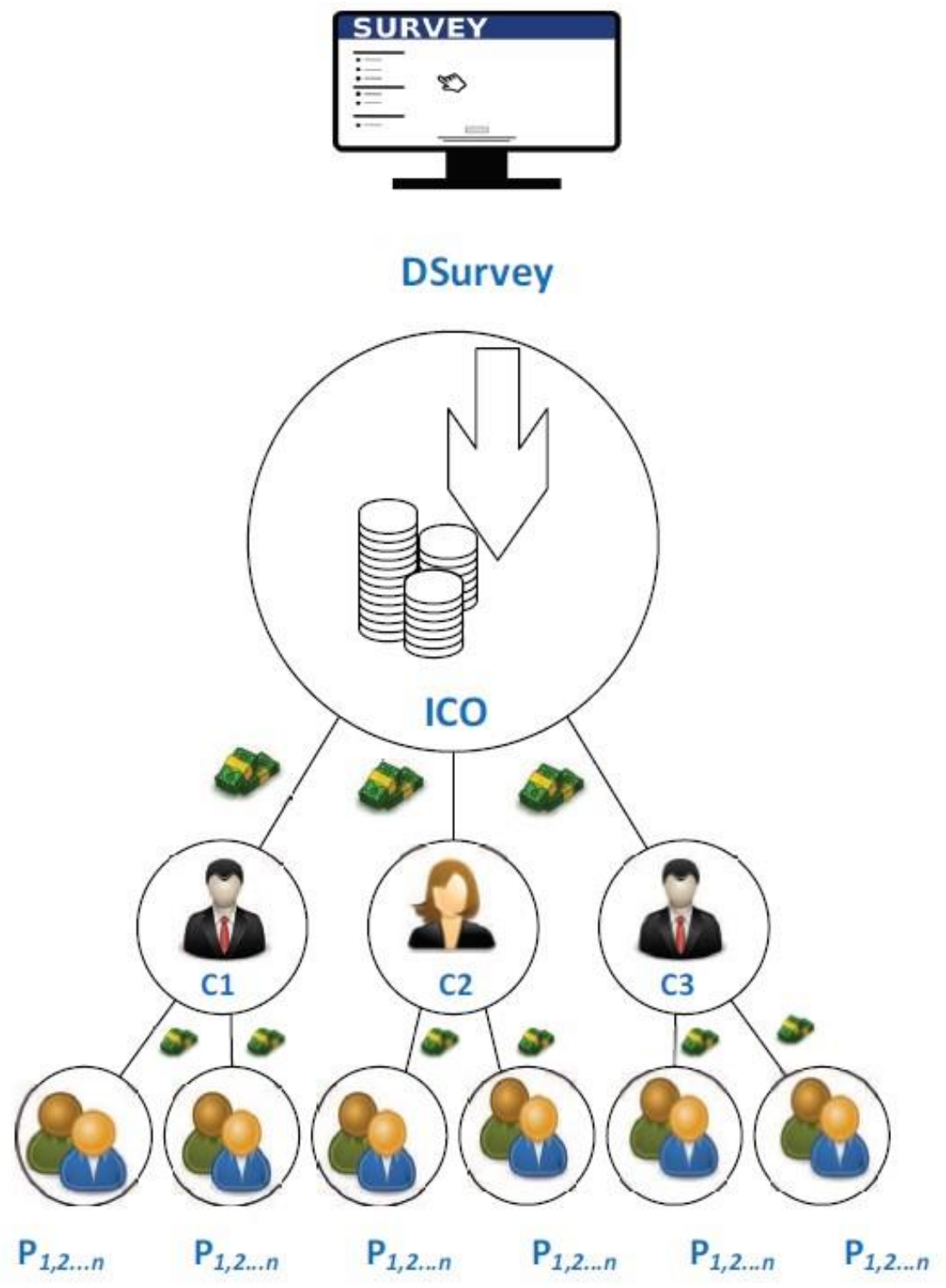

Figure 2. System architecture of D-Survey application.

\subsection{Functions of Framework}

Each peer in the system described in Figure 3 provides many functions to facilitate the survey process. These functions mainly include initiating an ICO, registering, validating a client or 
participant, uploading the survey questionnaire, and update the results. The process of each peer is explained as follows:

- Initiating an ICO: An ICO is offered by the survey application in the start of the process. It is initiated to start the economy so that the clients can buy the coins/tokens in order to join the system. These tokens can be evenly distributed among the joined clients to be fair.

- Survey deployed by clients: A client creates and publishes it. The content of the survey is made public on the ledger while the answers are kept private. Only the client who is the owner of the survey has the authority to read the answers.

- Survey performed by participants: Participants register and log onto the system, they are verified in a decentralized manner after satisfying the defined requirements set by the survey creator. The answers are stored on the private ledger R1. After clients receive the results of the survey, participants will be rewarded with the bounty.

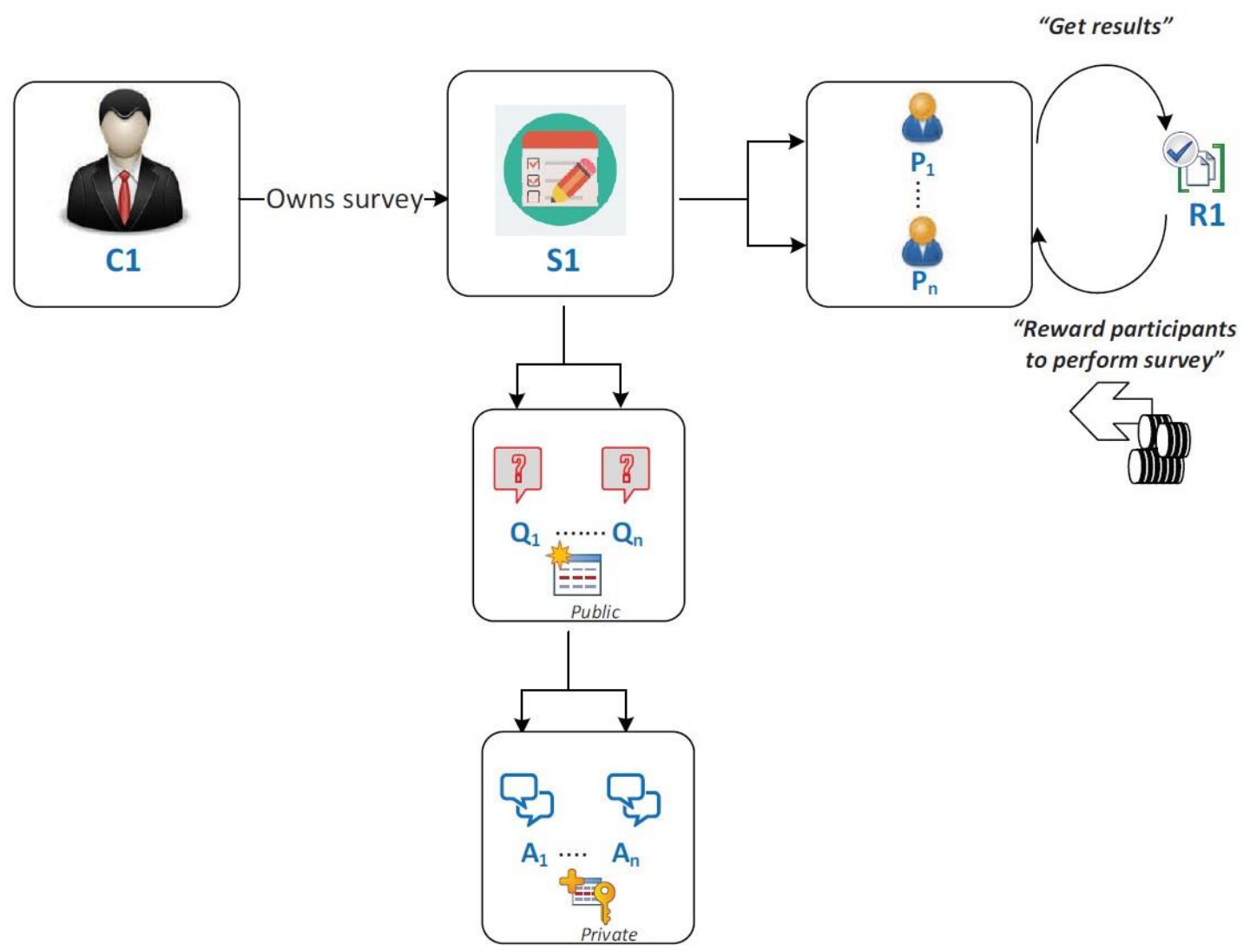

Figure 3. Illustration of DSurvey workflow.

DSurvey - the service provider, (1) creates an ICO and sells these coins to companies (C) who (2) will create surveys made up of questions and attach a bounty - made up of the bought coins - to these, and an encryption key. (3) Registered users, following the KYC (know your customer) process, are able to attempt the survey. (4) The bounty is passed onto the user. (5) The answer is encrypted using the survey provider's key and uploaded onto the system. The original company (C) makes the data available for sale. (6) Other companies can use the system to create a new survey or query the existing one. (7) When another company buys the existing data, a royalty fee is passed to the original creator of that data and (8) the list of users of the data is updated. 


\subsubsection{Incentive mechanism}

As described above, our platform is based on incentivising both the company as well as the participants. Companies own the surveys and are incentivised based on their ability to gather and resell data, participants are incentivised by providing their data (answer the surveys). The main reason behind incentivising is to justify and satisfy the stakeholders as per their given inputs. The same is described in Figure 4. For a survey to be done, client $\mathrm{C} 1$ makes micropayments in the form of tokens to the participants involved in the survey. Since the questions are public in our system, another client say C2 can read them and ask the owner of that survey to get answers regarding them. To avoid data manipulation, we proposed that $\mathrm{C} 2$ has to pay in order to get the asked results from $\mathrm{C} 1$. Only then, he can get the answers from R1 and from which the incentives are shared between $\mathrm{C} 1$ and the users whose data is being provided to the $\mathrm{C} 2$. Similarly, when $\mathrm{C} 2$ sells the data to $\mathrm{C} 3$, the price $\mathrm{C} 3$ pays to the $\mathrm{C} 2$ is shared as an incentive between $\mathrm{C} 1, \mathrm{C} 2$, and the users. Here, $\mathrm{C} 1$ is the creator while $\mathrm{C} 2$ and $\mathrm{C} 3$ are the clients and late adopters as mentioned in Figure 5.

3) $\mathrm{C} 2$ gets the desired

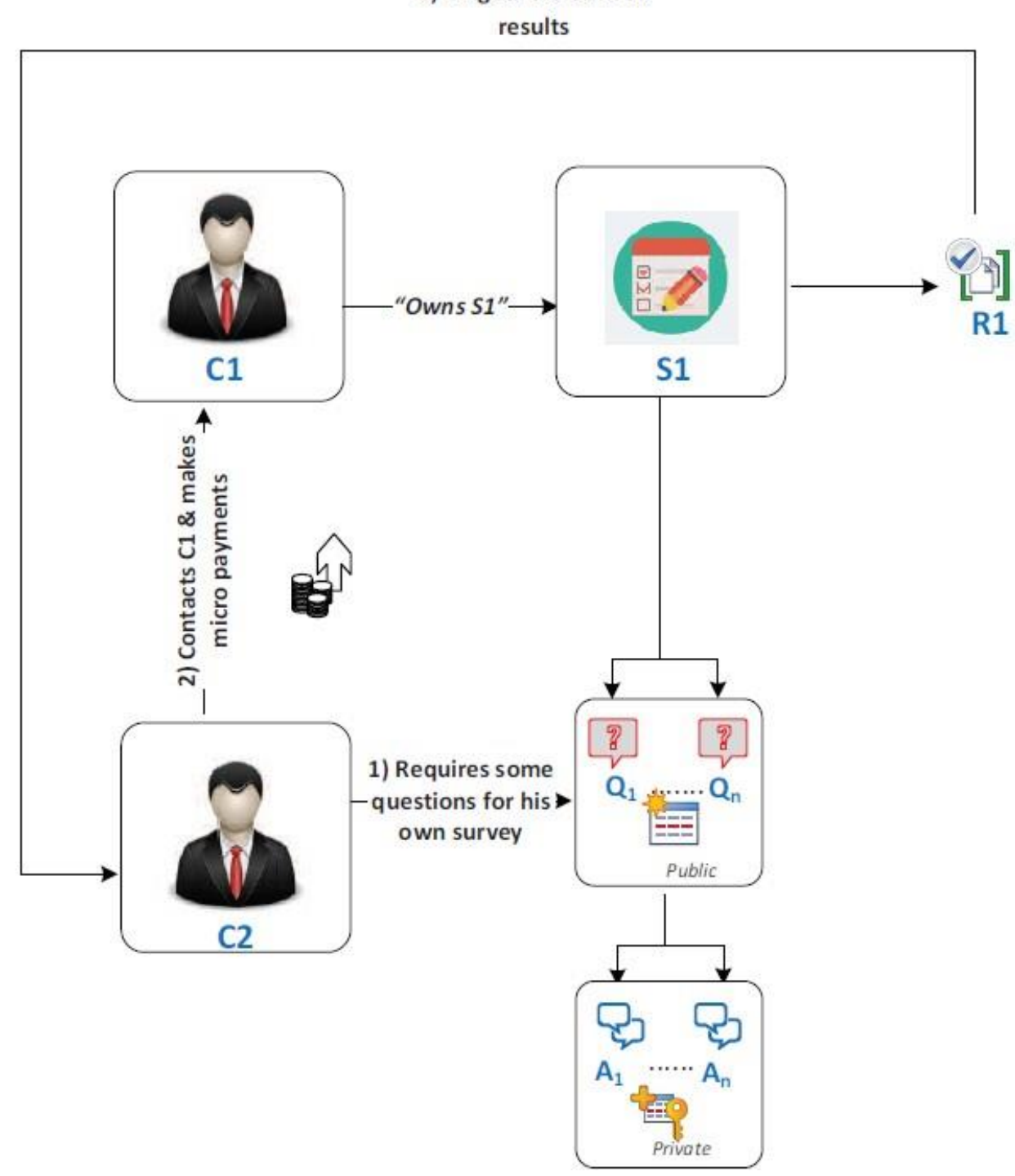

Figure 4. Survey results purchase process. 


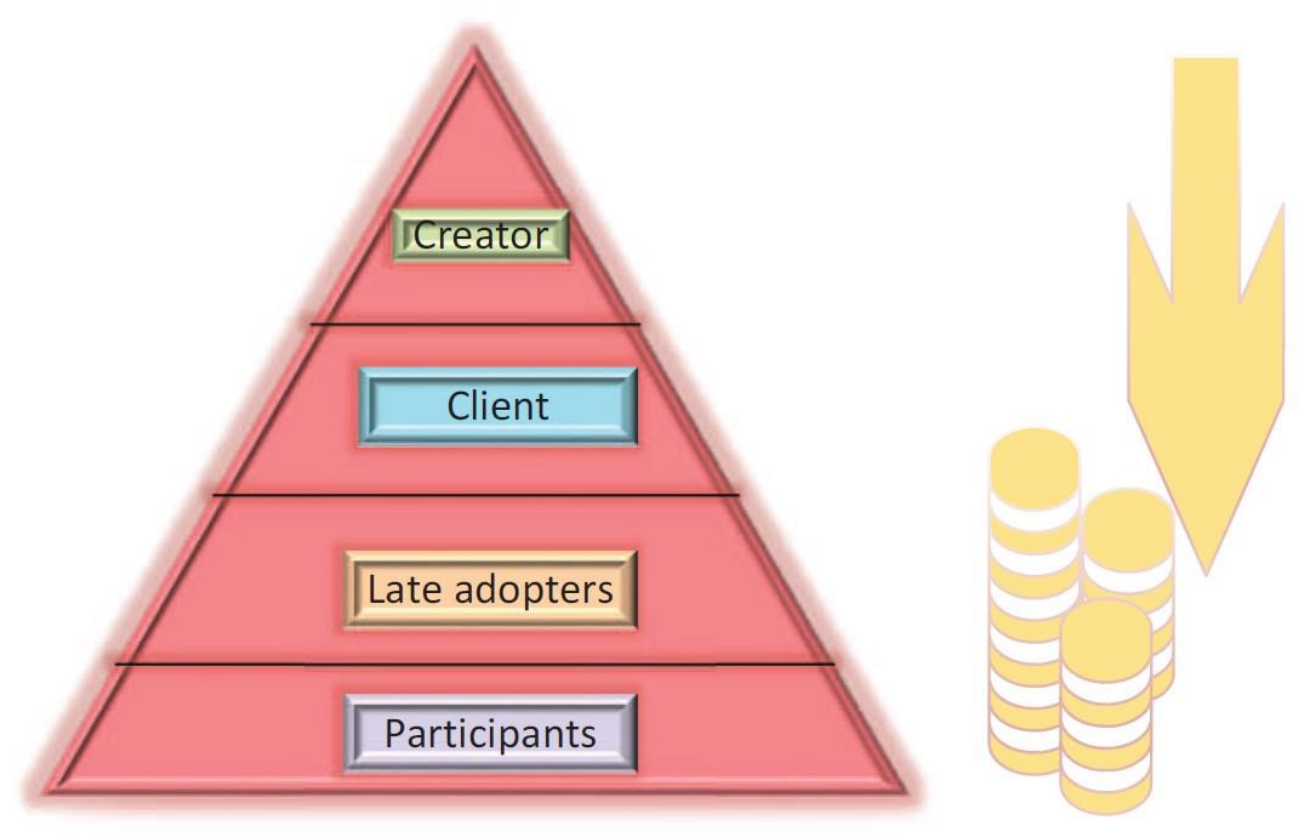

Figure 5. Pyramid incentive workflow.

Through our incentive mechanism, clients gain the financial benefit, and it avoids the unauthorized data sharing between two or more clients. This creates a collaborative hostile environment which ensures a system of implicit trust.

In our incentive mechanism, all the clients are paying the same amount of money to the parent companies irrespective of their position in the pyramid. All the late adopters won't be provided with any incentives after a certain no of companies own the data, as they will only be consumers of that data, hence they will only be able to buy it.

\subsubsection{Reward mechanism}

Despite the authors of [13] have pointed out rewards bring negative effects in most scenario regarding the organization environment, the research also claimed that rewards can be used as facilitation for knowledge sharing. Other research [13-16] suggested that rewards, to some extent, influence the willingness of an individual to share their knowledge. Both [13] and [15] clearly indicates the role of rewards in increasing engagements which is what reward mechanism aims to achieve.

The reward mechanism is introduced to guarantee the incentivising attribute of the platform. The reason behind this reward mechanism is that stakeholders should be rewarded fairly for their inputs [17]. Therefore, in the proposed incentivising-based platform, rewards are used as extrinsic incentives in exchange for desired inputs, and obviously, the better the rewards are, the more likely that valuable inputs will be provided. Moreover, fair rewards for all stakeholders of the economy will ensure an efficient economy with fair incentives which is the key to a successful system [17]. The reward mechanism in our system functions in the following main forms.

\subsubsection{Bounty}

By attempting a survey, participants will receive their rewards which are paid by the creator. This initial reward is the first incentive that participants receive after they complete the survey. By 
paying micro payments for participants attempting the survey, the survey creator encourages users to provide their data for an initial reward. Therefore, this can also be considered as a way to entice participants to share their data [17,18], so that the creator can get wide availability of results. However, participants have to go through the Know Your Customer process to register themselves against the system before they can attempt any surveys, thus, they can remain anonymous. The anonymity could be considered as a reward for users to go through the registration process. As rewarding transactions take place on a blockchain system to which participants are all connected, they can easily investigate the fairness of the share, thus, trust is ensured $[19,20]$. Further information regarding how this works will be demonstrated in section 5 .

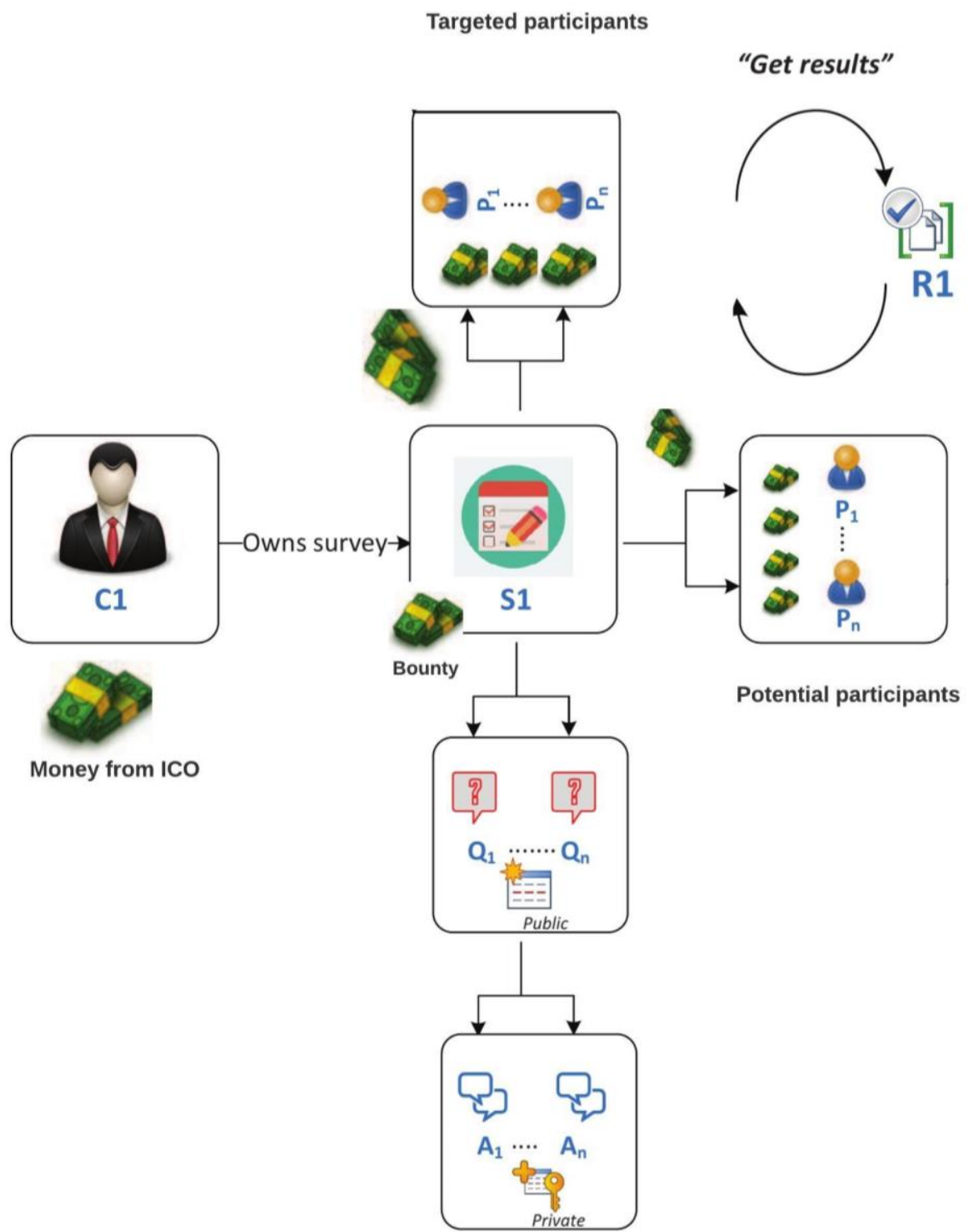

Figure 6. Bounty mechanism. 


\subsubsection{Royalty fee}

As discussed above, a royalty fee must be paid when a company resells the data. According to the incentive mechanism, when the data is resold, the money is shared with the original data creator, their participants and *the shared space. The royalty fee is considered as the data creator's reward for the creation of data. This is another incentive that participants receive. While [23] states that the motivation for sharing is increased when reward is more significant than cost but leave the question mark about the influence of reward systems on stimulation of one's knowledge sharing intention. The answer was proposed in [24] that extrinsic rewards positively influence ones' willingness to share. Details of how money is paid and shared among stakeholders will be discussed in section 5 .

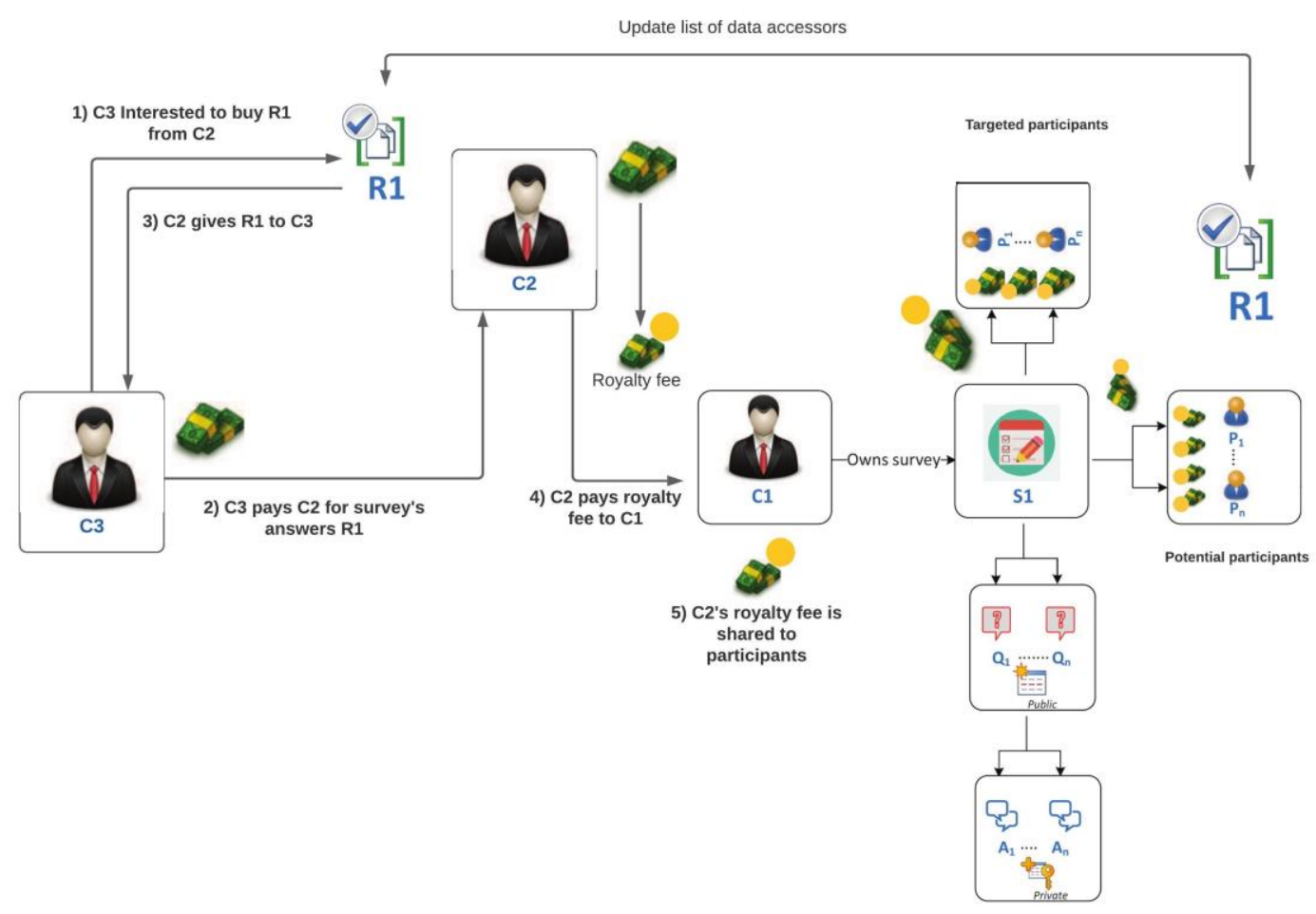

Figure 7. Royalty fee mechanism based on section 3.2.1's scenario.

\subsubsection{Early clients:}

The Shared Space: every time a new company buys the answers to the survey enters in a virtual shared space. This shared space gets a fee on every new transaction and this fee is divided among the participants of this space. In this way, every company after buying the survey can slowly get back the money invested. The money is splitted in the following way:

- Money is splitted every time a company enters the space in equal parts among the members.

- The company that created the survey gets counts as 2 (gets the double with reference to other companies) (the idea is to faster their ROI).

Given all these elements the earlier companies buy data the more money they get back (higher price for data and less company to split with the fee). 
This can be view as either a reward from the creator enticing other companies to become their "early clients" [6,8]; or the reward for the early clients themselves when they can join the shared space early and resell the data, this is similar to the idea discussed in [23] and [24]. Although the clients neither create nor generate data, they still get their rewards because of their early investments to buy the data and expose it to other companies which could lead to increase in sales as well as the widespread of the data. As a result, data creators can build up their reputation and all companies can create more connections with each other. From that point, this mechanism also shows its encouragement for companies in the system to share their data as "personal and community outcome expectations" are considered as important reason for knowledge sharing [69]. Through this reward mechanism, companies are encouraged to share their data with others. Moreover, this also stimulate companies in the system to generate new data and/or high-quality data for members of the system to use. And users are given rewards for giving out their data, so that companies can have a good source of data and learn their customers better.

Our reward mechanism does not only contribute to the environment some distinct attributes, but it also reinforces those proposed by the incentive mechanism. These reciprocal stimulations will promisingly create a competitive commercial environment where incentives will drive the platform to become a disruptive solution to the data economy with a new data ownership and privacy paradigms.

\section{SECURITY MODEL}

\subsection{Data ownership}

Since our goal is to give data ownership to the user itself, the survey data is created and stored on a public-permissioned ledger so that it provides high trust because the validator nodes are operated by a global network of trusted institutions. All of them are monitoring each other for good conduct. Smart contracts are designed to allow for safe record ownership, permission, and integrity. The contract's state-transition functions carry out policies, enforcing data alteration only by legitimate transactions. Such policies can be designed to implement any set of rules which govern a particular survey detail, as long as it can be represented computationally. For example, there are some specific rules which force third parties to obtain the consent from user and the owning companies before accessing their data.

\subsection{KYC and User Privacy}

Know your customer (KYC) is a big challenge while making a survey application. Every client wants to know about the individuals participating in the survey. In our system, the participants will have to register themselves through the 2 -stage KYC registration process. In the 1st-stage, the user creates a basic profile, while in the second level, they can include more data, such as their qualifications, these are safeguarded through the Sovrin Architecture. This allows the user to remain anonymous and to only reveal the personal details which can allow him to participate in the survey. DSurvey also creates a Participant Rank Index (PRI), based on their performance. The higher the PRI, higher the chances of attaining maximum surveys (financial benefits). Using PRI our application allows clients to target a specific audience. PRI not only increase engagement but also helps clients to understand their audience better. 


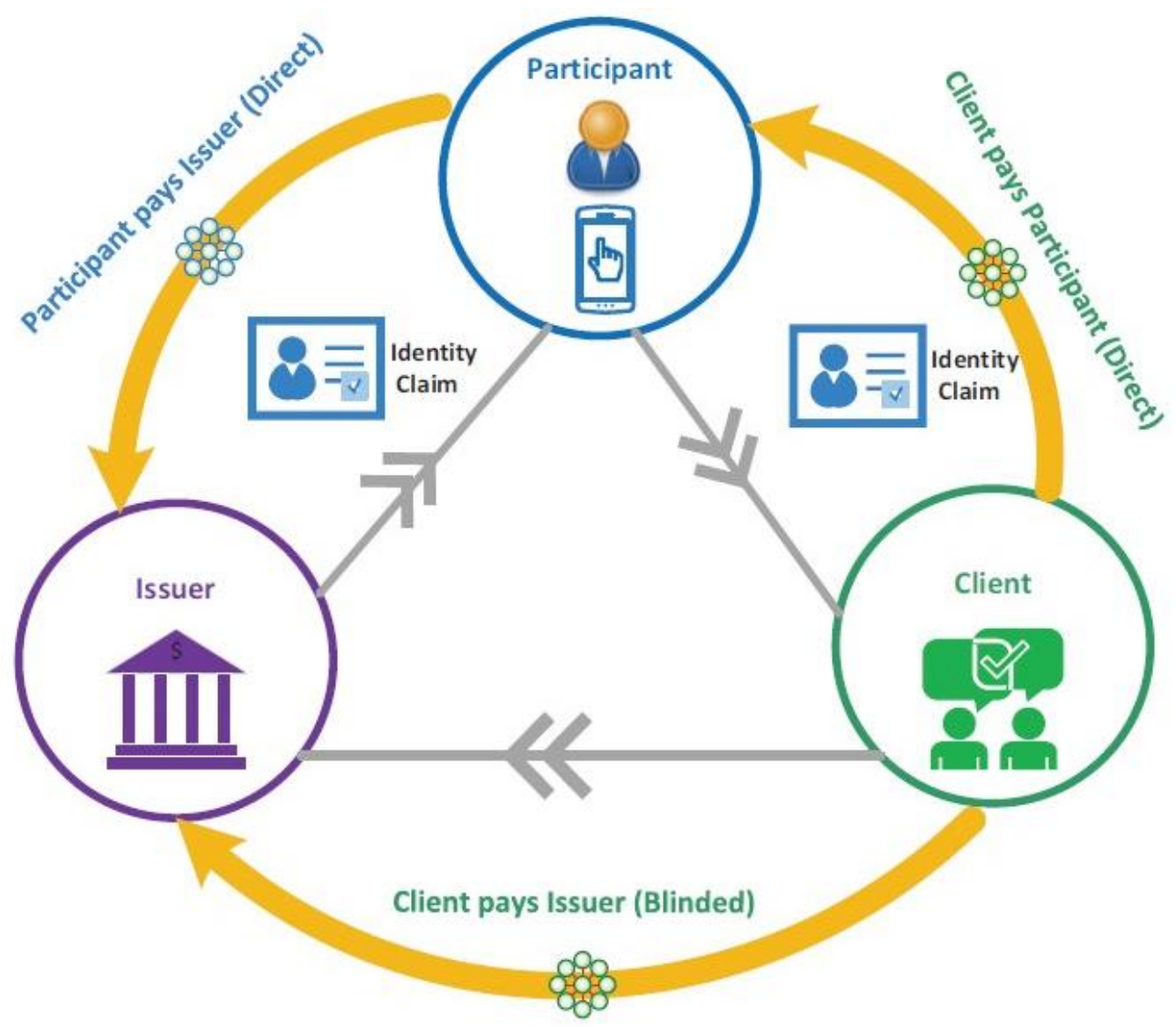

Figure 8. KYC through Sovrin architecture.

For KYC, we use the Sovrin architecture as described in Figure 8, using Sovrin tokens [25]. This architecture works in three steps:

- Step 1: Participant creates an identity claim through an issuer, using his already recorded trusted documents.

- Step 2: The claim is presented to the client in order to allow the participant to do the survey.

- Step 3: Client pays tokens to issuer of digital credentials either directly or blindly through participant.

\section{DEMONSTRATION}

DSurvey has been designed and implemented as a web application, the application itself has been utilized within Deakin University within a sand boxed controlled environment. The aim of it is to make the testers recognize the benefits of decentralised blockchain technology in the context of survey applications, and how they can benefit users. The experiment has provided us with both the user experience feedback and the knowledge for the constructing and improving the revenue stream here presented. The system's functionalities are the following:

- Step 1: Survey creation, with a bounty attached.

- Step 2: Survey reports with performance.

- Step 3: Survey Data Marketplace view.

- Step 4: Full history of all the data and ownership. 
As individual participants, they can gain tokens from both answering the survey royalties, with full history. Participant can gain tokens from both answering the survey and market transactions. The user interface of the application is showed in Figure 9 and Figure 10. Survey participants are able to track their answers usage with timestamp logs and view available surveys on the market (Figure 9).

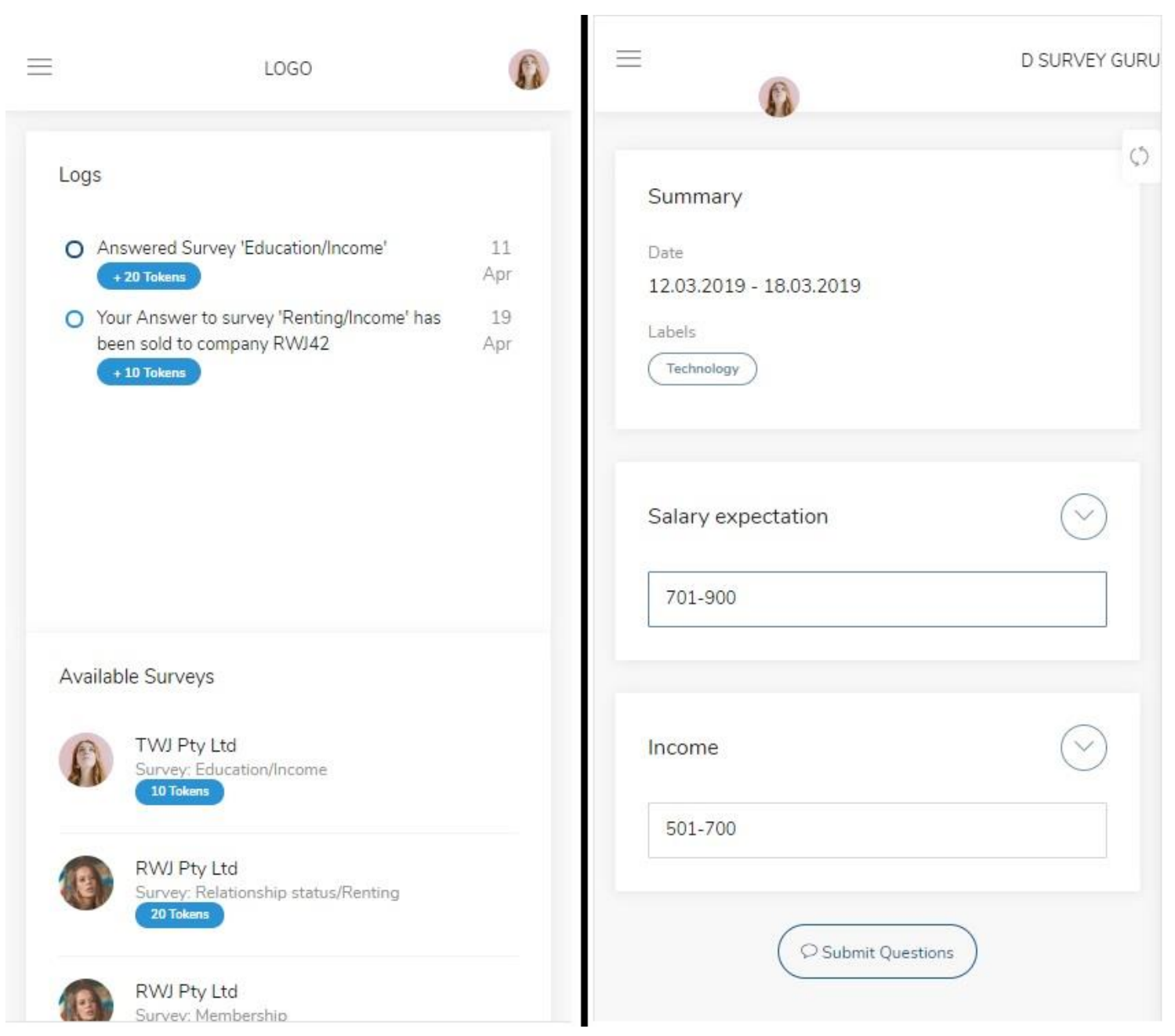

Figure 9. User answers tracking, available surveys on market (left) and summary report.

The survey result report view (Figure 10) with graphs and figures are presented to data generators (companies) for their analysis of survey's performance. By applying blockchain technology in managing transactions, a data timeline with timestamp and all other critical details are well presented (Figure 10). Therefore, data provenance is guaranteed to be transparent throughout the system. 


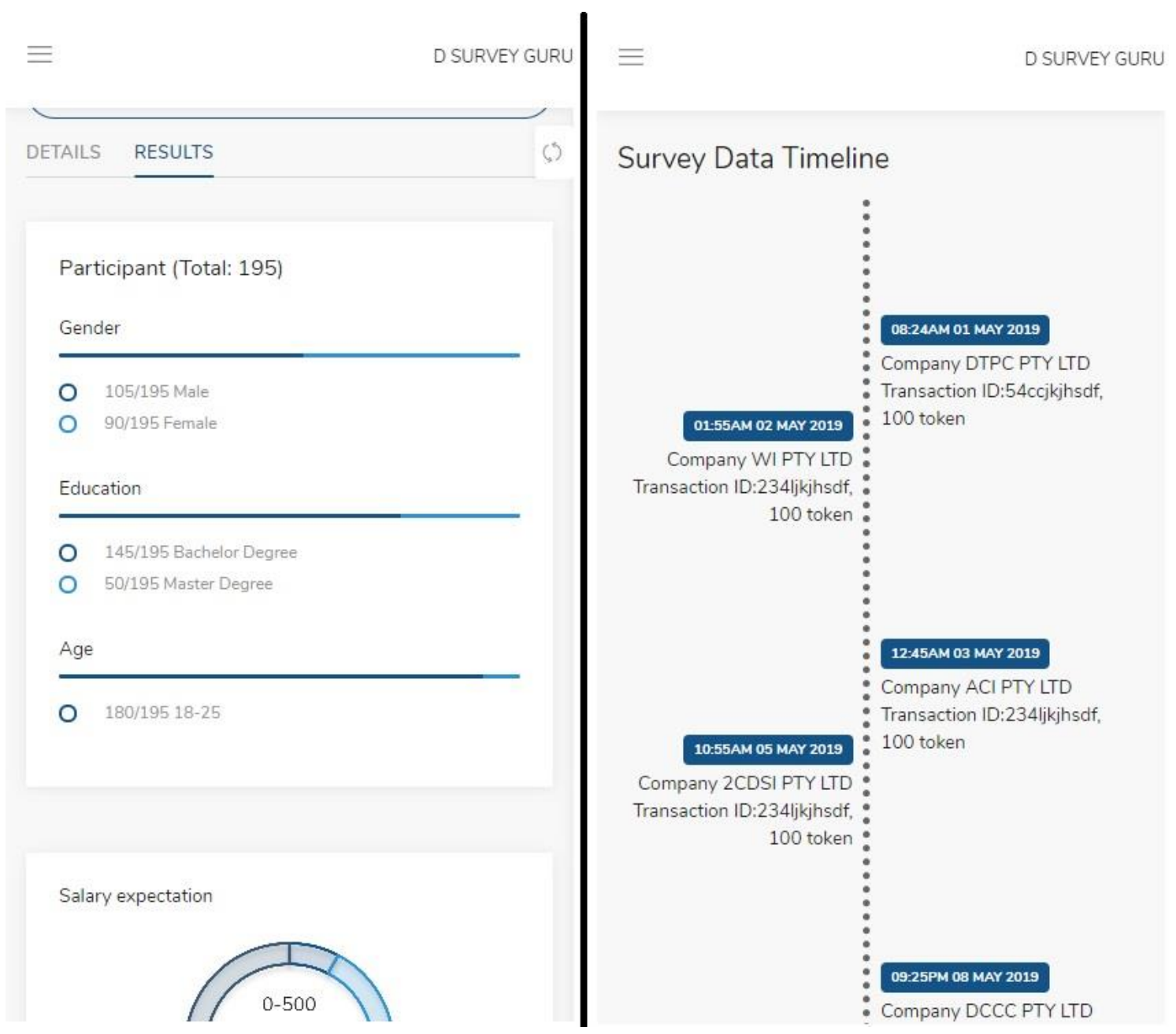

Figure 10. Survey performance (left) and data life tracking (data provenance).

\subsection{Revenue stream model simulation}

\subsubsection{The goal}

The goal of this revenue stream is to showcase the right balance between the following points: Having companies creating surveys to:

- Increase the amount of data available.

- Give higher fees to DSurvey.

Having companies exploiting already existing surveys to:

- Add values to users with recurrent fees (with marginal cost equal to 0).

- Help the ROI for company investing in creating new surveys.

Even though the surveys will be specific, this model also pushes companies to try to as much as possible to differentiate questions generating data that are not in the platform yet and are broad enough to be exploited by other companies (otherwise if data are already existing or too specific no one will buy the answer and the company will not have a ROI). This will lead to an organic 
data turnover where the outcome will be a platform full of data covering all topics and broad enough to be exploited by more than one entity.

To achieve these goals the revenue is created considering the need for fresh data and the risk aversion of each company. This model presents different strengths depending on the position of the customer.

For companies creating new surveys:

- Higher upfront costs.

- Higher possible ROI.

- $\rightarrow$ For risk takers.

For companies exploiting already existing surveys:

- Lower upfront costs

- Lower possible ROI

- $\rightarrow$ For risk averse companies.

\subsubsection{Economic returns over time}

The following shows the return of investment for participating in the system, with every step formulating a different aspect.

Time 0: identifies a moment where no answers to specific required questions are present in the platform and uses do not own answers yet.

- When a company needs to do a survey it pays 0,12USD per question per user.

- COMPANY1 creates the survey and pay for the answers.

- This amount is shared among DSurvey (15\%) and the user (85\%).

- Every user gets $0.10 / \mathrm{q} \rightarrow \sim 30 \%$ higher than market average.

- Every company pays $0,12 / \mathrm{q} / \mathrm{u} \rightarrow \sim 30 \%$ lower than market average creating a clear winwin situation for both parties.

Time 1: identifies a time where a company can decide to create a new survey or use existing ones.

- When a company wants to do a survey, it can check on the DSurvey platform to see if some of the questions they will ask to have already been asked. Our Time 1 simulates that another company has already created the survey (or part of it), so COMPANY2 can buy the "second hand" answers for $0.09 / \mathrm{q} / \mathrm{u}$.

- COMPANY2 buys answers of the survey created by COMPANY1. This amount is shared among DSurvey (81\%), the user (151\%) and the virtual shared space* (77\\%).

Time n: Identifies any moment there after where a company wants to acquire existing data.

- Every time a new company buys the survey, it pays the price paid by the previous company minus $0,002 \%$ (this to slowly decrease the price of data in order to adjust it with the freshness of the data) and if the price reaches the $70 \backslash \%$ of the initial price it stops decreasing. Then, any new companies will get a fixed price at $701 \%$ if they want to purchase the data. (this to prevent companies to buy old data for free). 
- COMPANY buys answer of the survey created by COMPANY1 at the adjusted price. This amount, as in the time step 1, is shared among DSurvey $(8 \%)$, the user $(15 \%)$ and the shared space $(77 \%)$.

\begin{tabular}{|c|c|c|c|}
\hline \multirow[t]{2}{*}{ Legends: } & \multicolumn{2}{|c|}{$\begin{array}{l}\text { Price new question } \\
\text { Price old question } \\
\text { Number of exploits }\end{array}$} & \multirow{2}{*}{$\begin{array}{l}0.12 \\
0.09 \\
\text { STION }\end{array}$} \\
\hline & $\begin{array}{c}\text { NEW QUESTION } \\
\text { FORMULA }\end{array}$ & $\begin{array}{r}\text { EXPLOIT QU } \\
\text { FORMU }\end{array}$ & \\
\hline $\begin{array}{l}\text { COMPANY COST (CC) } \\
\text { FIRST COMPANY FEE } \\
\text { COMPANY FEE } \\
\text { USER FEE } \\
\text { DSURVEY FEE }\end{array}$ & $\begin{array}{l}P N Q \\
0 \\
0 \\
C C * 0,85 \\
C C * 0,15\end{array}$ & $\begin{array}{l}\left(\frac{0.998}{1}\right)^{n}(P O \\
\sum_{x=1}^{n} 0.77\left(C C_{x}\right)\left(\frac{P O}{x}\right. \\
\sum_{x=n E n t r y}^{n} 0.77\left(C C_{x}\right) \\
\sum_{x=1}^{n} 0.15\left(C C_{x}\right) \\
\sum_{x=1}^{n} 0.08\left(C C_{x}\right)\end{array}$ & 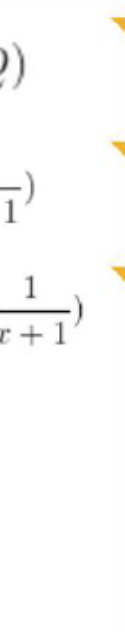 \\
\hline
\end{tabular}

Figure 11. Fees calculation formulas.

\section{Discussion AND CONCLUSION}

Our experiment provided a simulation environment to showcase the capabilities of our framework and the completeness of our application. The revenue stream simulation proved that the application could compete with the existing platforms and provide a return of investment for both companies and end users higher than market price. Besides, it is important to understand that moving forward, data will become more and more important, and as we have seen, also matter of legal contentions. With the advent of the blockchain, Web 3.0 will revolutionize how data is perceived as we will be able to truly own our data, know where it is and who is using it, inevitably shaping the terms privacy and ownership into new forms.

Moreover, DSurvey shows the potential to be a replacement for the current online surveys by solving the problem of low response rates and lack of trust from participants. Data, thus, will be considered as a new kind of commodity which can be own and sell for profit. With the participant of data-driven companies and the development of data monetization processes, a decentralized 
network with implicit trust and robust security features is definitely a sustainable platform for a multi-billion dollars economy to flourish.

Our system aims to be one in a line of more applications which will provide users with true ownership of their data, giving them the choice of what to do with it. Especially in the advent of Distributed AI where our data will not only be a tool for creating personal financial benefits, but also to contribute to the creation of powerful artificial intelligence based on personal high quality health data.

However, this paper did not evaluate on the performance and scalability of the system. Therefore, future research might need to focus on performance evaluation of the system to showcase its processing speed, transactions per time unit, computational power requirements, and more. Besides, scalability of the system should also be assessed to verify its feasibility.

\section{REFERENCES}

[1] Magnus, A. Detecting Survey Fraud by applying the Relative Difference Score to Survey Response Time. 2017.

[2] A. Opher, A. Onda, A. Chou, and K. Sounderrajan, The Rise of the Data Economy: Driving Value $\begin{array}{lllll}\text { through Internet of Things Data } & \text { Monetization. }\end{array}$ https://www.ibm.com/downloads/cas/4JROLDQ7. (2016).

[3] The United Nations. Data Economy: Radical transformation or dystopia?. Frontier Technology Quarterly. content/uploads/sites/45/publication/FTQ_1_Jan_2019.pdf. (2019).

[4] B. Marr, The 7 most data-rich companies in the world. World Economic Forum https://www.weforum.org/agenda/2015/02/the-7-most-data-rich-companies-in-the-world/. (2015).

[5] 2017. Hyperledger. https://www.hyperledger.org. (2017).

[6] Couper, M. P. Web surveys - A review of issues and approaches, Public Opining Quarterly. no. 64:464-494. (2000).

[7] Couper, M. P., M. W. Traugott, and M. J. Lamias. Web survey design and administration. Public Opinion Quarterly. no. 65:230-253. (2001).

[8] Dommeyer, C. J., and E. Moriarty. Comparing two forms of an e-mail survey: Embedded vs attached. International Journal of Market Research no. 42:39-50. (2000).

[9] PwC Russia. Personal data as a commodity. Protect.me. https://www.pwc.ru/en/publications/assets/protect-me.pdf. (2018).

[10] S. Nakamoto, Bitcoin: A peer-to-peer electronic cash system, 2008.

[11] Alshalali, T., M'Bale, K. and Josyula, D. Security and Privacy of Electronic Health Records Sharing Using Hyperledger Fabric. City, 2018.

[12] Jiang, S., Cao, J., Wu, H., Yang, Y., Ma, M. and He, J. BlocHIE: A BLOCkchain-Based Platform for Healthcare Information Exchange. City, 2018.

[13] Wu, H., Cao, J., Yang, Y., Tung, C. L., Jiang, S., Tang, B., Liu, Y., Wang, X. and Deng, Y. Data Management in Supply Chain Using Blockchain: Challenges and a Case Study. City, 2019.

[14] A. Tobin, and D. Reed, The inevitable rise of self-sovereign identity. The Sovrin Foundation, 29, 2016.

[15] Bock, G.-W. and Kim, Y.-G. Breaking the Myths of Rewards: An Exploratory Study of Attitudes about Knowledge Sharing. IRMJ, 15 (04/01 2002), 14-21.

[16] Chai, S. and Kim, M. What makes bloggers share knowledge? An investigation on the role of trust. International Journal of Information Management, 30, 5(2010/10/01/ 2010), 408-415.

[17] Hsu, M.-H., Ju, T. L., Yen, C.-H. and Chang, C.-M. Knowledge sharing behavior in virtual communities: The relationship between trust, self-efficacy, and outcome expectations. International Journal of Human-Computer Studies, 65, 2 (2007/02/01/2007), 153-169.

[18] Kankanhalli, A., Tan, B. C. Y. and Wei, K.-K. Contributing Knowledge to Electronic Knowledge Repositories: An Empirical Investigation. MIS Quarterly, 29, 1 (2005),113-143.

[19] Symeonidis, I., Schroers, J., Mustafa, M. A. and Biczók, G. Towards Systematic Specification of Non-Functional Requirements for Sharing Economy Systems. City,2019. 
[20] Shrestha, A. K., Joshi, S. and Vassileva, J. Customer Data Sharing Platform: A Blockchain-Based Shopping Cart. City, 2020.

[21] Shrestha, A. K. and Vassileva, J. User Data Sharing Frameworks: A Blockchain-Based Incentive Solution. City, 2019.

[22] Zheng, Z., Peng, Y., Wu, F., Tang, S. and Chen, G. ARETE: On Designing Joint Online Pricing and Reward Sharing Mechanisms for Mobile Data Markets. IEEE Transactions on Mobile Computing, 19, 4 (2020), 769-787.

[23] Chiu, C.-M., Hsu, M.-H. and Wang, E. T. G. Understanding knowledge sharing in virtual communities: An integration of social capital and social cognitive theories. Decision Support Systems, 42, 3 (2006/12/01/ 2006), 1872-1888.

[24] Wei, C., Chang, Y., Wang, W., Chou, H. and Chen, K. Factors that Influence Sharing Behaviors in Sharing Economy Based on the Theory of Social Capital and Social Exchange: Example of TaiwanBased USPACE. City, 2019.

[25] 2018. SovrinTM: A Protocol and Token for Self-Sovereign Identity and Decentralized Trust. https://sovrin.org/wp content/uploads/Sovrin-Protocol-and-Token-White-Paper.pdf (2018)

\section{ACKNOWLEDGEMENTS}

The authors would like to thank everyone who contributed to this paper!

(C) 2021 By AIRCC Publishing Corporation. This article is published under the Creative Commons Attribution (CC BY) license. 